\title{
Synthesis and in vitro antibacterial and antifungal evaluation of quinoline analogue azetidin and thiazolidin derivatives
}

\author{
Shraddha M. Prajapati ${ }^{a}$, Rajesh H. Vekariya ${ }^{a}$, Kinjal D. Patel ${ }^{a}$, \\ Shyamali N. Panchal ${ }^{a}$, Hitesh D. Patel ${ }^{a, *}$, Dhanji P. Rajani ${ }^{b}$, Smita Rajani ${ }^{b}$ \\ ${ }^{a}$ Department of Chemistry, School of Sciences, Gujarat University, Ahmedabad, Gujarat, India \\ ${ }^{b}$ Microcare Laboratories and Tuberculosis Research Center, Surat, Gujarat, India \\ *Tel.: +91-079-26300969; Fax: +91-079-26308545 \\ *E-mail address: drhiteshpatel1@gmail.com
}

\begin{abstract}
A library of quinoline analog two novel series of azetidin $\left(\mathrm{SH}_{1-5}\right)$ and thiazolidin $\left(\mathrm{SH}_{\mathrm{a}-\mathrm{e}}\right)$ derivatives were designed and synthesized with simple and eco-friendly methodologies. The structures of the compounds were elucidated with the aid of elemental analysis, IR, ${ }^{1} \mathrm{H}-\mathrm{NMR}$ and mass spectral data. These novel synthesized compounds were evaluated for antibacterial activity against two grampositive bacteria (Staphylococcus aureus, Staphylococcus pyogenus) and two gram-negative bacteria (Pseudomonas aeruginosa, Escherichia coli). The title compounds were also studied for their antifungal activity with Candida albicans, Aspergillus niger, Aspergillus clavatus using the broth dilution technique. Most of the compounds were the best bio-active desired antibacterial analog with less MIC value against different tested strains.
\end{abstract}

Keywords: Antibacterial; Antifungal; Quinoline; azetidin derivatives; thiazolidin derivatives

\section{INTRODUCTION}

Quinoline derivatives in general are known to have a broad range of applications in medicinal, bio-organic, industrial as well as in the fields of synthetic organic chemistry. Their derivatives have been found to possess various biological activities like anti-malarial, antibacterial, anti-fungal, anti-asthmatic, antihypertensive, anti-inflammatory and anti-platelet activity [1]. They also exhibit anti-tubercular and immune depressant activities [2]. Few promising compounds with the quinoline ring system like, pamaquine (1), chloroquine (2), tafenoquine (3), bulaquine (4), quinine (5) and mefloquine (6) as an antimalarial agent and amodiaquine (7) as an antimalarial as well as anti-inflammatory agent (Figure 1) [3-5]. 
<smiles>CCN(CC)CCCC(C)Nc1cc(OC)cc2cccnc12</smiles>

1<smiles>COc1cc(C)c2c(Cc3cccc(C(F)(F)F)c3)c(OC)cc(NC(C)CCCN)c2n1</smiles>

3<smiles>CCN(CC)CCCC(C)Nc1cccc2cc(Cl)cnc12</smiles>

2<smiles>CCC1=CC(NC(C)CCCN=C(C)C2CCOC2=O)c2ncccc2C1</smiles>

4<smiles>CCN(CC)Cc1cc(Nc2ccnc3cc(Cl)ccc23)ccc1O</smiles>

6<smiles>OC(c1cc(C(F)(F)F)nc2c(C(F)(F)F)cccc12)C1CCCCN1</smiles>

Figure 1. Few promising compounds with quinoline ring system. 
Quinoline or 1-aza-napthalene or benzo pyridine is one of the most nitrogen containing heterocyclic aromatic compound having a variety of applications in medicinal, bioorganic, industrial as well as in the fields of synthetic organic chemistry, becomes reason to pick out quinoline as N-heterocyclic compound for our study (Figure 2). Moreover, in recent years there are greater societal expectations that chemists should produce greener and more sustainable chemical processes. So we have tried to follow green methods for the synthesis, as far as possible. During our literature for the synthesis of quinoline derivatives, Nafees Ahmed et al. studied structural activity relationship of quinoline-2,4-diols for anti-HIV activity and they also conclude that the activity is due to unsubstituted ring $\mathrm{B}$ and two free - $\mathrm{OH}$ groups [6].<smiles>Oc1cc(O)c2ccccc2n1</smiles><smiles>O=c1cc(O)c2ccccc2[nH]1</smiles>

Figure 2. Resonating structure of quinoline-2,4-diol.

Therefore, we found quinoline-2,4-diol having hydroxyl group at $2^{\text {nd }}$ and $4^{\text {th }}$ positions will became quite interesting for the study. We also tried to incorporate the azetidin-2-one moiety and thiazolidin-4-one moiety in quinoline-2,4-diol scaffold to enhance its activity.

Azetidinones have received considerable attention during last two decades as they are endowed with a variety of biological activities and have a wide range of therapeutic activities (Figure 3). The $\beta$-lactam ring is the main feature of the most of the penicillins and other antibiotics. The $\beta$-lactam ring shows various biological activities such as antifungal $[7,8]$, antibacterial [9,10], antitubercular [11,12], anticonvulsant [13], analgesic, anti-inflammatory [14], synthetic precursor for amino acids [15], antiviral [16] and many more.<smiles>O=C1C(Cl)C([Al])N1c1nnc(COc2c(Cl)cc(Cl)cc2Cl)s1</smiles>

4-argio-3-chloro-1-(5-((2,4,6-trichlorophenoxy)methyl)-

1,3,4-thiadiazol-2-yl)azetidin-2-one

Antibacterial 
<smiles>[R]C1C(Cl)C(=O)N1Nc1ccc([N+](=O)[O-])cc1[N+](=O)[O-]</smiles>

\section{4-substituted-3-chloro-1-((2,4-dinitrophenyl) amino)azetidin-2-one \\ Anticonvulsant}

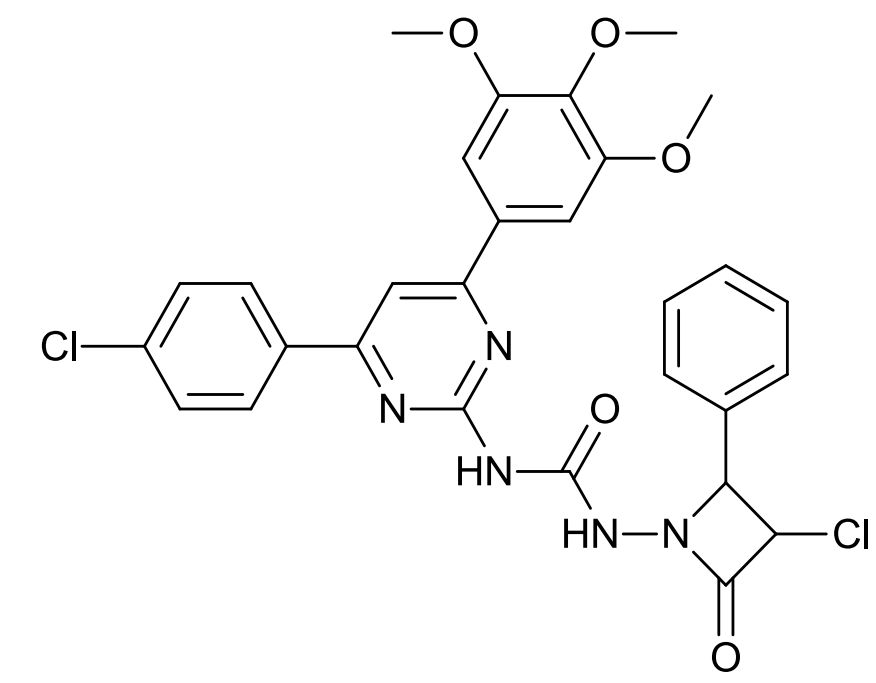

1-[4-(4-chloro-phenyl)-6-(3,4,5-trimethoxyphenyl)pyrimidin2-yl-ureido]-3-chloro-4-aryl-2-azetidinones

Antitubercular

\section{1}

Figure 3. Biologically Potent Azetidinones derivatives.

Variety of heterocyclic products including drugs [17, 18], dyes and intermediates such as thiazol yellow, thioflavin T., thidiazuron [19], herbicides [20], insecticides [21,22] and many more attributed to possess thiazolidin-4-one.

Besides the above applications, thiazolidinones moiety is also associated with a broad spectrum of biological activities including antibacterial [23,24], antifungal [25], antiinflammatory [26,28], hypnotic, anticonvulsant, antitubercular [29], antiviral [30,31], antihistamines, anthelmintic, cardiovascular and anticancer [32]. 
<smiles>COc1cc(C2SCC(=O)N2CC(=O)c2ccncc2)ccc1O</smiles>

2-(4-hydroxy-3-methoxyphenyl)-3-(2-oxo-2-(pyridin-4-yl) ethyl)thiazolidin-4-one Antimycobecterial<smiles>CN(C)CCCN1C(=O)CSC1c1c(F)cccc1F</smiles>

2-(2,6-difluorophenyl)-3-(3-(dimethylamino) propyl)thiazolidin-4-one Antihistamine

13<smiles>[R][Y]1ccc(C2SCC(=O)N2NC(=O)COc2ccc([R])cc2)cc1</smiles>

Substituted-N-(4-oxo-2-phenylthiazolidin-3-yl)

-2-( $p$-tolyloxy)acetamide

Antimicrobial

14

Figure 4. Some biologically potent thiazolidin-4-one derivatives.

Quinolines could be synthesized using various methodologies and recently we have reported one review article on it [33]. Due to the environmental pollution, we have tried to carry out all the transformations using green chemistry principle. 


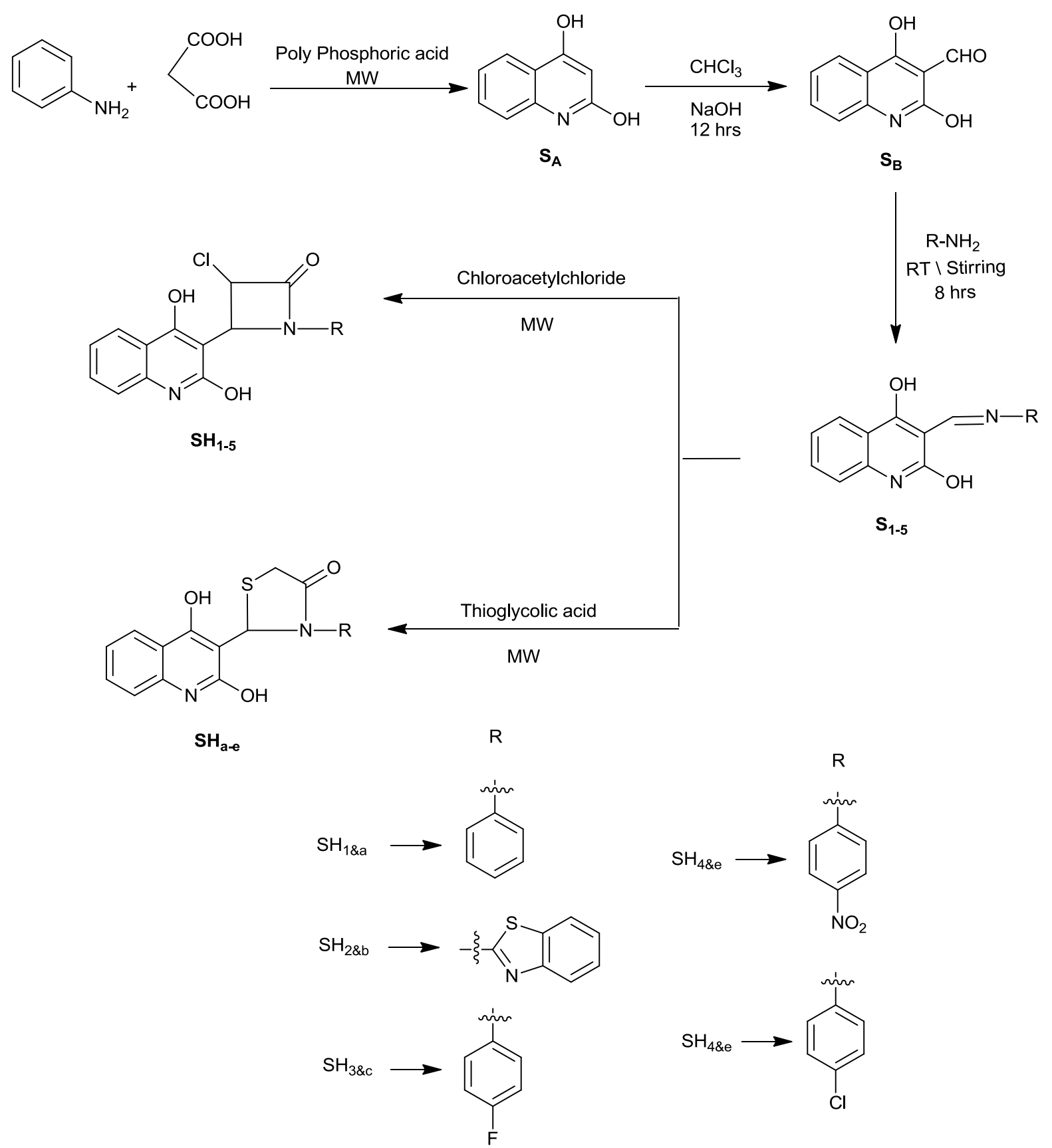

Scheme 1. Synthetic route for quinoline analog azetidin $\left(\mathrm{SH}_{1-5}\right)$ and thiazolidin $\left(\mathrm{SH}_{\mathrm{a}-\mathrm{e}}\right)$ derivatives.

We have used microwave irradiation for this multi-step synthesis. In view of the need to discover potent novel antimicrobial agents, we have synthesized novel quinoline 2,4-diol analogue 2-azitidinone/ thiazolidin-4 one derivatives (Figure 5) and evaluated their antimicrobial and antifungal activity against different strains of bacteria and fungal using the broth micro dilution method and compared with some reference drugs. 

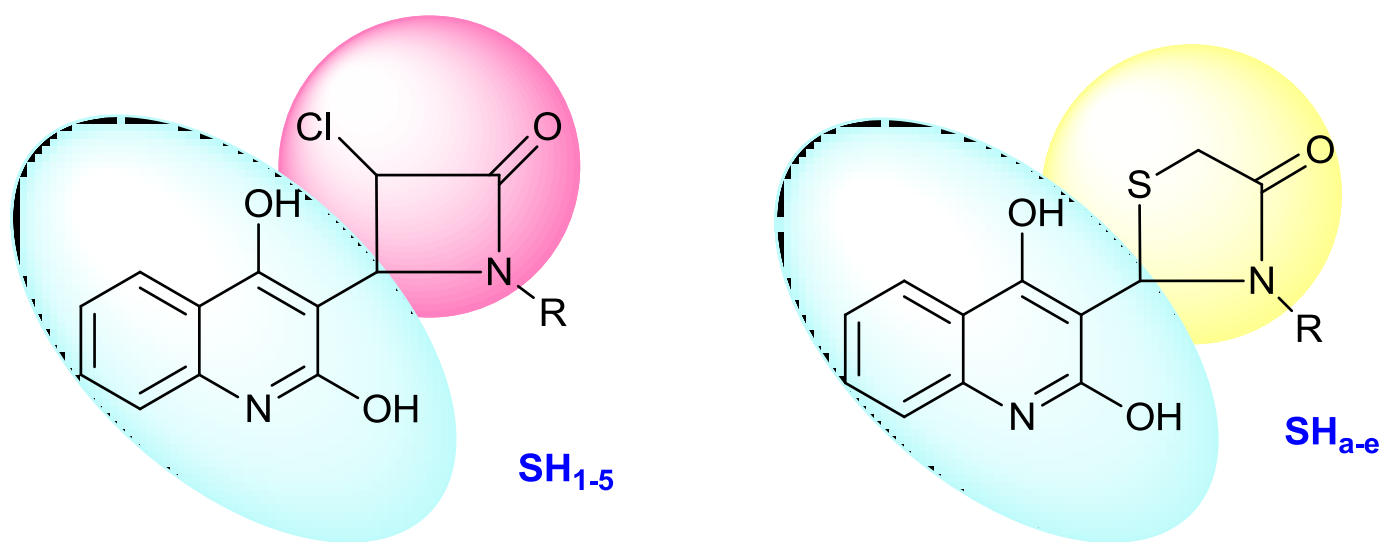

Figure 5. Quinoline analog 2-azitidinone/ thiazolidin-4-one derivatives.

\section{MATERIALS AND METHODS}

All Starting materials and other reagents were purchased from commercial suppliers and were used without any further purification unless otherwise indicated. The reactions were assayed by thin layer chromatography (TLC) and terminated as judged by the consumption of starting material. Analytical thin-layer chromatography (TLC) was performed on silica gel G 60 F254 (Merck) plates and eluted with the appropriate solvent ratios (v/v). The melting points were recorded in optimelt automated melting point system and were uncorrected. IR spectra were recorded on a Perkin-Elmer 377 spectrophotometer, ${ }^{1} \mathrm{H}$ NMR spectra was measured in Bruker AV $400 \mathrm{MHz}$ using $\mathrm{CDCl} 3$ as a solvent and TMS as an internal standard. Mass spectra was recorded on Advion Expression CMS, USA, using Methanol: Water: Formic acid (80: 20: 0.1) as mobile phase. Elemental analysis was performed on the Vario MICRO cube, elementary CHN analyzer serial no.: 15084053. Here we have used the CEM Discover microwave system for synthesis. Its model no.: 908010 and made CEM Matthews. Inc, USA.

\section{1. General procedure for the synthesis of 4 -Hydroxyquinolin-2(1H)-one $\left(\mathrm{S}_{\mathrm{A}}\right)$.}

Aniline $(7 \mathrm{~mL}, 5 \mathrm{mmol})$ and malonic acid $(5.2 \mathrm{~g}, 5 \mathrm{mmol})$ was thoroughly mixed with $20 \mathrm{~g}$ poly phosphoric acid (PPA) $\left\{\mathrm{P}_{2} \mathrm{O}_{5}(287.9 \mathrm{~g})\right.$ was added to $85 \%$ phosphoric acid $(200 \mathrm{~g}$, $118.4 \mathrm{~mL}$ ) with stirring and microwave heating. The mixture was then heated for next 15 min. $\}$ in $250 \mathrm{~mL}$ flask and heated with stirring in a microwave reactor (CEM-S-discover) at $400 \mathrm{~W}$ during $2 \times 20 \mathrm{~min}$ with $5 \mathrm{~min}$ interval. The temperature reached $210{ }^{\circ} \mathrm{C}$. Then the mixture was poured into crushed ice and a cream colored solid was filtered. The crude product was purified by extraction with $\mathrm{EtOH}$ and a white crystalline analytical sample was obtained [21].

Yield- $79 \%$; m.p. $340{ }^{\circ} \mathrm{C}$.; IR ( $v$ max cm-1): $3277 \mathrm{~cm}^{-1}, 1728 \mathrm{~cm}^{-1}, 1308.8 \mathrm{~cm}^{-1}, 1630$ $\mathrm{cm}^{-1}, 1600 \mathrm{~cm}^{-1}, 687 \mathrm{~cm}^{-1}, 725 \mathrm{~cm}^{-1}, 660.8 \mathrm{~cm}^{-1}, 625.2 \mathrm{~cm}^{-1}$; 1H NMR (400 MHz, DMSO-d6, $\delta \mathrm{ppm}):(1 \mathrm{H}, \mathrm{d}) 7.85,(1 \mathrm{H}, \mathrm{t}) 7.79,(1 \mathrm{H}, \mathrm{t}) 7.55,(1 \mathrm{H}, \mathrm{d}) 8.23,(1 \mathrm{H}, \mathrm{s}) 5.35,(1 \mathrm{H}, \mathrm{s}) 6.23,(1 \mathrm{H}, \mathrm{s})$ 11.53; ESI-MS (m/z): $161.05(\mathrm{M})^{+}$; Elemental analysis: Cal. C, 67.09; H, 4.36; N, $8.69 \%$. Found. C, 67.19; H, 4.24; N, 8.70\%. 


\section{2. General Procedure for the Synthesis of 4-Hydroxy-3-formyl-quinolin-2(1H)-one \\ $\left(\mathbf{S}_{\mathrm{B}}\right)$}

Sodium hydroxide ( $80 \mathrm{ml}$ of $15 \%)$ was charged with 0.0124 mole of 4hydroxyquinolin-2(1H)-one and was cooled to $4{ }^{\circ} \mathrm{C}$ and stirred. Subsequently the temperature inside the flask was maintained at $80{ }^{\circ} \mathrm{C}$ on a water bath. At $80{ }^{\circ} \mathrm{C}, 40 \mathrm{~mL}(0.5 \mathrm{~mole})$ of chloroform was introduced in three portions at intervals of fifteen minutes down the condenser on hot water bath. Stirring was continued for 12 hours, and then the reactionmixture was cooled to RT. The orange colored liquid was acidified with dilute sulfuric acid. It was extracted with ethyl acetate and dried with anhydrous sodium sulfate. Ethyl acetate was removed under vacuum and the solid obtained was purified by column chromatography $\left(\mathrm{SiO}_{2}\right)$ in hexane, ethyl acetate $(7: 3)$ mixture.

Yield- $79 \%$; m.p. $>350^{\circ} \mathrm{C}$; IR (v max cm-1): $1730 \mathrm{~cm}^{-1}, 770 \mathrm{~cm}^{-1}, 712 \mathrm{~cm}^{-1}, 1629 \mathrm{~cm}^{-}$ $1,1288 \mathrm{~cm}^{-1}, 1300 \mathrm{~cm}^{-1}, 1107 \mathrm{~cm}^{-1}$; 1H NMR (400 MHz, DMSO-d6, $\left.\delta \mathrm{ppm}\right):(1 \mathrm{H}, \mathrm{d}) 7.89$, $(1 \mathrm{H}, \mathrm{t}) 7.63,(1 \mathrm{H}, \mathrm{t}) 6.98,(1 \mathrm{H}, \mathrm{d}) 9.03,(1 \mathrm{H}, \mathrm{s}) 5.55,(1 \mathrm{H}, \mathrm{s}) 6.32,(1 \mathrm{H}, \mathrm{s}) 10.97,(1 \mathrm{H}, \mathrm{s}) 9.73$; ESI-MS (m/z): $189.4(\mathrm{M})^{+}, 190.3(\mathrm{M}+1)^{+}$; Elemental analysis: Cal. C, 63.46; H, 3.74; N, 7.42 \%. Found. C, 63.56; H, 3.77; N, $7.43 \%$.

\section{3. General Procedure for the Synthesis of 4-Hydroxy-3-formylideneamino-quinolin- 2(1H)-one $\left(S_{1}-S_{5}\right)$}

To a solution quinoline aldehyde $(1 \mathrm{mmol})$ in dichloromethane and various aromatic anilines $(1 \mathrm{mmol})$ was added into the reaction mixture. It was stirred for 8 hours at room temperature. The reaction mixture was poured into ice-water to afforded crude products which was purified by either crystallization in ethanol or purified by column chromatography using pet ether and ethyl acetate $(9: 1)$ as eluent. They are conformed by spectral as well as analytical analysis.

\section{(Z)-3-((phenylimino)methyl)quinoline-2,4-diol $\left(\mathrm{S}_{1}\right)$}

Yellow colored solid compound, M.P. $267-269{ }^{\circ} \mathrm{C}$, Yield 92\%; Spectral data: IR (KBr) $3269.34 \mathrm{~cm}^{-1}, 1622.04 \mathrm{~cm}^{-1}, 684.22 \mathrm{~cm}^{-1}$; Mass: $264.09\left(\mathrm{M}^{+}\right), 265.09(\mathrm{M}+1)^{+}$; ${ }^{1} \mathrm{H}$ NMR: $\delta_{\mathrm{H}}(400 \mathrm{MHz}, \mathrm{DMSO}):(1 \mathrm{H}, \mathrm{s}) 10.64,(1 \mathrm{H}, \mathrm{s}) 5.03,(1 \mathrm{H}, \mathrm{s}) 7.65,(1 \mathrm{H}, \mathrm{m}) 7.72,(1 \mathrm{H}, \mathrm{t}) 7.43$, $(1 \mathrm{H}, \mathrm{d}) 8.13,(1 \mathrm{H}, \mathrm{s}) 8.78,(2 \mathrm{H}, \mathrm{d}) 6.97,(2 \mathrm{H}, \mathrm{m}) 7.35,(1 \mathrm{H}, \mathrm{m}) 6.83$; Elemental Analysis: $\mathrm{C}$, $72.68 ; \mathrm{H}, 4.60 ; \mathrm{N}, 10.62$.

\section{3-(((6-methoxybenzo[d]thiazol-2-yl)imino)methyl)quinoline-2,4-diol $\left(\mathbf{S}_{2}\right)$}

Brown colored solid compound, M.P. $>300{ }^{\circ} \mathrm{C}$, Yield $85 \%$; Spectral data: IR (KBr) $1615.70 \mathrm{~cm}^{-1}, 3065.28 \mathrm{~cm}^{-1}, 1038.16 \mathrm{~cm}^{-1}, 1265.62 \mathrm{~cm}^{-1}$; Mass: $351.07\left(\mathrm{M}^{+}\right), 352.09(\mathrm{M}+1)^{+}$; ${ }^{1} \mathrm{H}$ NMR: $\delta_{\mathrm{H}}(400 \mathrm{MHz}, \mathrm{DMSO}):(1 \mathrm{H}, \mathrm{s}) 10.42,(1 \mathrm{H}, \mathrm{s}) 4.98,(1 \mathrm{H}, \mathrm{s}) 7.73,(1 \mathrm{H}, \mathrm{m}) 7.81,(1 \mathrm{H}$, t) $7.49,(1 \mathrm{H}$, d) $8.69,(1 \mathrm{H}, \mathrm{s}) 9.01,(1 \mathrm{H}, \mathrm{d}) 6.98,(1 \mathrm{H}, \mathrm{d}) 7.48,(1 \mathrm{H}, \mathrm{d}) 7.43,(3 \mathrm{H}, \mathrm{s}) 5.33$; Elemental Analysis: Cal. C, 61.51; H, 3.74; N, 11.97 \%. Found. C, 61.52; H, 3.77; N, 11.87 $\%$.

\section{(Z)-3-(((4-fluorophenyl)imino)methyl)quinoline-2,4-diol (S3)}

Dark yellow colored solid compound, M.P. $287-288^{\circ} \mathrm{C}$, Yield 84\%; Spectral data: IR (KBr) $3076.46 \mathrm{~cm}^{-1}, 1613.54 \mathrm{~cm}^{-1}, 690.52 \mathrm{~cm}^{-1}$; Mass: $282.09\left(\mathrm{M}^{+}\right), 283.08(\mathrm{M}+1)^{+} ;{ }^{1} \mathrm{H}$ NMR: $\delta_{\mathrm{H}}(400 \mathrm{MHz}, \mathrm{DMSO}):(1 \mathrm{H}, \mathrm{s}) 10.49,(1 \mathrm{H}, \mathrm{s}) 5.23,(1 \mathrm{H}, \mathrm{m}) 7.84,(1 \mathrm{H}, \mathrm{m}) 7.97,(1 \mathrm{H}, \mathrm{t})$ 7.23, $(1 \mathrm{H}, \mathrm{d}) 7.99,(1 \mathrm{H}, \mathrm{s}) 8.38,(2 \mathrm{H}, \mathrm{d}) 6.99,(2 \mathrm{H}, \mathrm{d}) 7.34$; Elemental Analysis: Cal. C, 68.04; H, 3.95; N, $9.94 \%$. Found. C, 68.08; H, 3.85; N, $9.98 \%$. 


\section{(Z)-3-(((4-nitrophenyl)imino)methyl)quinoline-2,4-diol (S4)}

Dark yellow colored solid compound, M.P. $>300{ }^{\circ} \mathrm{C}$, Yield $89 \%$; Spectral data: IR (KBr) $3067.51 \mathrm{~cm}^{-1}, 1621.07 \mathrm{~cm}^{-1}, 875.09 \mathrm{~cm}^{-1}$; Mass: $309.07\left(\mathrm{M}^{+}\right), 310.08(\mathrm{M}+1)^{+}$; ${ }^{1} \mathrm{H}$ NMR: $\delta_{\mathrm{H}}(400 \mathrm{MHz}, \mathrm{DMSO}):(1 \mathrm{H}, \mathrm{s}) 10.53,(1 \mathrm{H}, \mathrm{s}) 4.98,(1 \mathrm{H}, \mathrm{s}) 7.32,(1 \mathrm{H}, \mathrm{m}) 7.48,(1 \mathrm{H}, \mathrm{t})$ 7.27, $(1 \mathrm{H}, \mathrm{d}) 8.38,(1 \mathrm{H}, \mathrm{s}) 8.72$, $(2 \mathrm{H}, \mathrm{d}) 6.92,(2 \mathrm{H}, \mathrm{d}) 8.21$; Elemental Analysis: Cal. C, 62.18; $\mathrm{H}, 3.56$; N, $13.57 \%$. Found. C, 62.14; H, 3.48; N, $13.59 \%$.

\section{(Z)-3-(((4-chlorophenyl)imino)methyl)quinoline-2,4-diol (S5)}

Light yellow colored solid compound, M.P. $265-266{ }^{\circ} \mathrm{C}$, Yield $94 \%$; Spectral data: IR (KBr) $3081.00 \mathrm{~cm}^{-1}, 1618.79 \mathrm{~cm}^{-1}, 870.18 \mathrm{~cm}^{-1}$; Mass: $298.05\left(\mathrm{M}^{+}\right), 300.10(\mathrm{M}+1)^{+} ;{ }^{1} \mathrm{H}$ NMR: $\delta_{\mathrm{H}}(400 \mathrm{MHz}, \mathrm{DMSO}):(1 \mathrm{H}, \mathrm{s}) 10.62,(1 \mathrm{H}, \mathrm{s}) 5.28,(1 \mathrm{H}, \mathrm{s}) 7.68,(1 \mathrm{H}, \mathrm{m}) 7.73,(1 \mathrm{H}, \mathrm{t})$ 7.34, $(1 \mathrm{H}, \mathrm{d}) 8.03,(1 \mathrm{H}, \mathrm{s}) 8.94,(2 \mathrm{H}, \mathrm{d}) 6.87,(2 \mathrm{H}, \mathrm{d}) 7.62$; Elemental Analysis: Cal. C, 64.35; H, 3.69; N, $11.86 \%$. Found. C, 64.38; H, 3.74; N, $11.82 \%$.

\section{4. General Procedure for the Synthesis of 3-chloro-4-(2,4-dihydroxyquinolin-3-yl)-1- azetidin-2-one ( $\left.\mathrm{SH}_{1}-\mathrm{SH}_{5}\right)$}

To the solution of Schiff base (1 mmol) in dimethylformamide (DMF), triethylamine (TEA) $(1.2 \mathrm{mmol})$ and chloroacetyl chloride $(1 \mathrm{mmol})$ were added, the reaction mixture was stirred properly and then place it at $300 \mathrm{~W}$ under microwave irradiation. Progress of the reaction was checked by TLC (Ethyl acetate/Toluene, 2:1). After completion of the reaction mixture was poured into ice-water. The crude product was obtained by filtration and crystallized in methanol. They are confirmed by spectral as well as analytical analysis.

\section{3-chloro-4-(2,4-dihydroxyquinolin-3-yl)-1-phenylazetidin-2-one $\left(\mathrm{SH}_{1}\right)$}

Dark brown colored solid compound, M.P. $>300{ }^{\circ} \mathrm{C}$, Yield $72 \%$; Spectral data: IR (KBr)3275.38 $\mathrm{cm}^{-1}, 1673.46 \mathrm{~cm}^{-1}, 1627.04 \mathrm{~cm}^{-1}, 684.22 \mathrm{~cm}^{-1}$; Mass: $340.4(\mathrm{M}+), 265.2$ (Base peak); ${ }^{1} \mathrm{H}$ NMR: $\delta_{\mathrm{H}}(400 \mathrm{MHz}, \mathrm{DMSO}):(1 \mathrm{H}, \mathrm{s}) 12.53,(2 \mathrm{H}, \mathrm{d}) 8.81,(2 \mathrm{H}, \mathrm{d}) 7.99,(1 \mathrm{H}, \mathrm{t})$ 7.61, $(1 \mathrm{H}, \mathrm{m}) 7.35,(1 \mathrm{H}, \mathrm{m}) 7.27,(1 \mathrm{H}, \mathrm{m}) 7.27,(1 \mathrm{H}, \mathrm{m}) 7.18,(1 \mathrm{H}, \mathrm{m}) 7.18,(1 \mathrm{H}, \mathrm{d})$ 5.94, $(1 \mathrm{H}$, s) 5.49, $(1 \mathrm{H}$, d) 5.24.; Elemental Analysis: Cal. C, 63.44; H, 3.85; N, $8.22 \%$. Found. C, 63.54; H, 3.88; N, $8.21 \%$.

\section{3-chloro-4-(2,4-dihydroxyquinolin-3-yl)-1-(6-methoxy-1,3-benzothiazol-2-yl)azetidin-2- one $\left(\mathrm{SH}_{2}\right)$}

Brown colored solid compound, M.P. $260{ }^{\circ} \mathrm{C}$, Yield $75 \%$; Spectral data: IR (KBr) $3259.52 \mathrm{~cm}^{-1}, 1671.43 \mathrm{~cm}^{-1}, 1632.45 \mathrm{~cm}^{-1}, 1035.42 \mathrm{~cm}^{-1} \& 1245.62 \mathrm{~cm}^{-1}$; Mass: 427.04 $(\mathrm{M}+) ;{ }^{1} \mathrm{H}$ NMR: $\delta_{\mathrm{H}}(400 \mathrm{MHz}, \mathrm{DMSO}):(1 \mathrm{H}, \mathrm{s}) 12.65,(2 \mathrm{H}, \mathrm{d}) 8.85,(2 \mathrm{H}, \mathrm{d}) 7.89,(1 \mathrm{H}$, t) 7.67, $(1 \mathrm{H}, \mathrm{m}) 7.34,(1 \mathrm{H}, \mathrm{m}) 7.28,(1 \mathrm{H}, \mathrm{m}) 7.28,(1 \mathrm{H}, \mathrm{m}) 7.12,(1 \mathrm{H}, \mathrm{m}) 7.12$, (1H, d) 5.97, $(1 \mathrm{H}, \mathrm{s}) 5.51,(1 \mathrm{H}, \mathrm{d}) 5.24$; Elemental Analysis: C, 56.14\%; H, 3.30\%; N, 9.82\%.

\section{3-chloro-4-(2,4-dihydroxyquinolin-3-yl)-1-(4-fluorophenyl) azetidin-2-one $\left.\mathrm{SH}_{3}\right)$}

Off white colored solid compound, M.P. $275^{\circ} \mathrm{C}$, Yield $76 \%$; Spectral data: IR (KBr) $3269.34 \mathrm{~cm}^{-1}, 1683.86 \mathrm{~cm}^{-1}, 1622.12 \mathrm{~cm}^{-1}, 827.46 \mathrm{~cm}^{-1}$; mass: $358.06\left(\mathrm{M}+\right.$.); ${ }^{1} \mathrm{H} \mathrm{NMR}: \delta_{\mathrm{H}}$ (400 MHz, DMSO): $(1 \mathrm{H}, \mathrm{s})$ 12.77, $(2 \mathrm{H}, \mathrm{d}) 8.82$, $(2 \mathrm{H}, \mathrm{d}) 7.95,(1 \mathrm{H}, \mathrm{t}) 7.64,(1 \mathrm{H}, \mathrm{m}) 7.39$, $(1 \mathrm{H}, \mathrm{m}) 7.25,(1 \mathrm{H}, \mathrm{m}) 7.25,(1 \mathrm{H}, \mathrm{m}) 7.14,(1 \mathrm{H}, \mathrm{m}) 7.14,(1 \mathrm{H}, \mathrm{d}) 5.90,(1 \mathrm{H}, \mathrm{s}) 5.54,(1 \mathrm{H}, \mathrm{d})$ 5.25.; Elemental Analysis: Cal. C, 60.26; H, 3.37; N, 7.81 \%. Found. C, 60.16; H, 3.29; N, $7.82 \%$. 


\section{3-chloro-4-(2,4-dihydroxyquinolin-3-yl)-1-(4-nitrophenyl) azetidin-2-one ( $\left.\mathrm{SH}_{4}\right)$}

Dark brown solid compound, M.P. $270{ }^{\circ} \mathrm{C}$, Yield- $75 \%$; Spectral data: IR (KBr) $3345.59 \mathrm{~cm}^{-1}, 1674.63 \mathrm{~cm}^{-1}, 1632.09 \mathrm{~cm}^{-1}, 840.51 \mathrm{~cm}^{-1}, 1516.05 \mathrm{~cm}^{-1}, 1319.31 \mathrm{~cm}^{-1}$; Mass: 385.05(M+); ${ }^{1} \mathrm{H}$ NMR: ${ }^{1} \mathrm{H}$ NMR: $\delta_{\mathrm{H}}(400 \mathrm{MHz}, \mathrm{DMSO}):(1 \mathrm{H}, \mathrm{s}) 12.79,(2 \mathrm{H}, \mathrm{d}) 8.84,(2 \mathrm{H}, \mathrm{d})$ 7.91, $(1 \mathrm{H}, \mathrm{t}) 7.67,(1 \mathrm{H}, \mathrm{m}) 7.42,(1 \mathrm{H}, \mathrm{m}) 7.22,(1 \mathrm{H}, \mathrm{m}) 7.22,(1 \mathrm{H}, \mathrm{m}) 7.13,(1 \mathrm{H}, \mathrm{m}) 7.13$, $(1 \mathrm{H}, \mathrm{d}) 5.84,(1 \mathrm{H}, \mathrm{s}) 5.59,(1 \mathrm{H}, \mathrm{d}) 5.21$; Elemental Analysis: Cal. C, 56.04; H, 3.14; N, 10.89 \%. Found. C, 56.12; H, 3.16; N, $10.92 \%$.

\section{3-chloro-1-(4-chlorophenyl)-4-(2,4-dihydroxyquinolin-3-yl) azetidin-2-one ( $\left.\mathrm{SH}_{5}\right)$}

Light brown coloured solid compound, M.P. $280^{\circ} \mathrm{C}$, Yield $75 \%$; Spectral data: IR (KBr) $3324.37 \mathrm{~cm}^{-1}, 1691.09 \mathrm{~cm}^{-1}, 1631.41 \mathrm{~cm}^{-1}, 837.31 \mathrm{~cm}^{-1}$;Mass: $374.02(\mathrm{M}+){ }^{1}{ }^{1} \mathrm{H}$ NMR: $\delta_{\mathrm{H}}(400$ MHz, DMSO): $(1 \mathrm{H}, \mathrm{s}) 12.72$, (2H, d) 8.79, $(2 \mathrm{H}, \mathrm{d}) 7.90,(1 \mathrm{H}, \mathrm{t}) 7.66,(1 \mathrm{H}, \mathrm{m}) 7.42,(1 \mathrm{H}, \mathrm{m})$ 7.22, (1H, m) 7.22, (1H,m ) 7.17, (1H,m ) 7.17, (1H, d) 5.94, (1H, s) 5.51, (1H, d) 5.20; Elemental Analysis: Cal. C, 57.62; H, 3.22; N, 7.47 \%. Found. Cal. C, 57.63; H, 3.24; N, 7.57 $\%$.

\section{5. General Procedure for the Synthesis of 2-(2, 4-dihydroxyquinolin-3-yl)-3-phenyl-1, 3-thiazolidin-4-one (SHa-e)}

To the solution of Schiff base (1 mmol) in dimethylformamide (DMF), thioglycolic acid $(1 \mathrm{mmol})$ and a drop of glacial acetic acid was added. The reaction mixture was heated under MW radiation at $300 \mathrm{~W}$. Progress of the reaction was checked by TLC (Ethyl acetate/Toluene, 2:1). After completion of the reaction, the reaction mixture was poured into ice water. Filter and collect the crude product and crystallized in methanol. They are confirmed by spectral as well as analytical analysis.

\section{2-(2,4-dihydroxyquinolin-3-yl)-3-phenyl-1,3-thiazolidin-4-one $\left(\mathrm{SH}_{\mathrm{a}}\right)$}

Dark brown color solid compound, M.P. $>300{ }^{\circ} \mathrm{C}$, Yield $71 \%$; Spectral data: IR(KBr) $1145.09 \mathrm{~cm}^{-1}, 1612.30 \mathrm{~cm}^{-1}, 1661.73 \mathrm{~cm}^{-1}, 3058.54 \mathrm{~cm}^{-1}$; Mass: $338.07(1 \mathrm{M}+) ;{ }^{1} \mathrm{H}$ NMR: $\delta_{\mathrm{H}}(400 \mathrm{MHz}, \mathrm{DMSO}):(1 \mathrm{H}, \mathrm{s}) 12.33,(1 \mathrm{H}, \mathrm{t}) 8.15,(1 \mathrm{H}, \mathrm{t}) 7.98,(1 \mathrm{H}, \mathrm{t}) 7.79,(1 \mathrm{H}, \mathrm{t})$ $7.58,(1 \mathrm{H}, \mathrm{m}) 7.49,(1 \mathrm{H}, \mathrm{m}) 7.42,(1 \mathrm{H}, \mathrm{m}) 7.28,(1 \mathrm{H}, \mathrm{m}) 7.21,(1 \mathrm{H}, \mathrm{s}) 5.17,(1 \mathrm{H}, \mathrm{s}) 3.82,(1 \mathrm{H}$, s) 3.75, $(1 \mathrm{H}, \mathrm{s}) 3.68$; Elemental Analysis: Cal. C, 63.89; H, 4.17; N, 8.28 \%. Found. C, 63.92; $\mathrm{H}, 4.19 ; \mathrm{N}, 8.22 \%$.

2-(2,4-dihydroxyquinolin-3-yl)-3-(6-methoxy-1,3-benzothiazol-2-yl)-1,3-thiazolidin-4-one $\left(\mathrm{SH}_{\mathrm{b}}\right)$

Off-white color solid compound, M.P. $250{ }^{\circ} \mathrm{C}$, Yield- $74 \%$; Spectral data: IR (KBr) $1151.98 \mathrm{~cm}^{-1}, 1615.70 \mathrm{~cm}^{-1}, 1668.06 \mathrm{~cm}^{-1}, 3065.28 \mathrm{~cm}^{-1}, 1038.16 \mathrm{~cm}^{-1} \& 1265.62 \mathrm{~cm}^{-1}$; Mass: $425.05(\mathrm{M}+)$; ${ }^{1} \mathrm{H}$ NMR: $\delta_{\mathrm{H}}(400 \mathrm{MHz}, \mathrm{DMSO}):(1 \mathrm{H}, \mathrm{s}) 12.34,(1 \mathrm{H}, \mathrm{t}) 8.30,(1 \mathrm{H}, \mathrm{t}) 7.89,(1 \mathrm{H}$, t) $7.78,(1 \mathrm{H}, \mathrm{t}) 7.57,(1 \mathrm{H}, \mathrm{m}) 7.49,(1 \mathrm{H}, \mathrm{m}) 7.38(1 \mathrm{H}, \mathrm{m}) 7.30,(1 \mathrm{H}, \mathrm{m}) 7.29,(1 \mathrm{H}, \mathrm{s}) 5.21,(1 \mathrm{H}$, s) $3.83,(1 \mathrm{H}, \mathrm{s}) 3.75,(1 \mathrm{H}, \mathrm{s}) 3.62$; Elemental Analysis: Cal. C, 56.46; H, 3.55; N, $9.88 \%$. Found. C, 56.56; H, 3.58; N, $9.89 \%$.

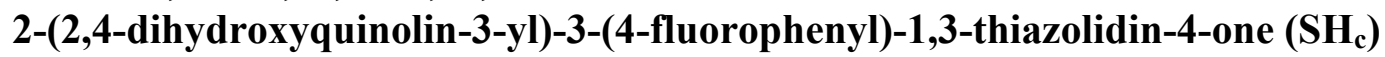

Brown coloured solid compound, M.P. $>300^{\circ} \mathrm{C}$ Yield- $75 \%$; Speactral data: IR(KBr) $1165.00 \mathrm{~cm}^{-1}, 1606.70 \mathrm{~cm}^{-1}, 1658.86 \mathrm{~cm}^{-1}, 3076.46 \mathrm{~cm}^{-1}, 867.97 \mathrm{~cm}^{-1}$; Mass: $356.06(\mathrm{M}+) ;{ }^{1} \mathrm{H}$ NMR: $\delta_{\mathrm{H}}(400 \mathrm{MHz}, \mathrm{DMSO}):(1 \mathrm{H}, \mathrm{s}) 12.23,(1 \mathrm{H}, \mathrm{t}) 8.21,(1 \mathrm{H}, \mathrm{t}) 7.91,(1 \mathrm{H}, \mathrm{t}) 7.84,(1 \mathrm{H}, \mathrm{t})$ 7.65, $(1 \mathrm{H}, \mathrm{m}) 7.57,(1 \mathrm{H}, \mathrm{m}) 7.40,(1 \mathrm{H}, \mathrm{m}) 7.32,(1 \mathrm{H}, \mathrm{m}) 7.23,(1 \mathrm{H}, \mathrm{s}) 5.15,(1 \mathrm{H}, \mathrm{s}) 3.99,(1 \mathrm{H}$, 
s) 3.81, $(1 \mathrm{H}, \mathrm{s}) 3.72$; Elemental Analysis: Cal. C, 60.67; H, 3.68; N, 7.86 \%. Found. Cal. C, $60.87 ; \mathrm{H}, 3.78 ; \mathrm{N}, 7.88 \%$.

\section{2-(2,4-dihydroxyquinolin-3-yl)-3-(4-nitrophenyl)-1,3-thiazolidin-4-one $\left(\mathrm{SH}_{\mathrm{d}}\right)$}

Dark brown colored solid compound, M.P. $>300{ }^{\circ} \mathrm{C}$,Yield- $73 \%$; Spectral data: IR (KBr) $1163.18 \mathrm{~cm}^{-1}, 1621.07 \mathrm{~cm}^{-1}, 1664.61 \mathrm{~cm}^{-1}, 3067.51 \mathrm{~cm}^{-1}, 875.09 \mathrm{~cm}^{-1}, 1562.34 \mathrm{~cm}^{-1}$, $1315.45 \mathrm{~cm}^{-1 ;}$ Mass: $383.06(\mathrm{M}+)$; ${ }^{1} \mathrm{H}$ NMR: $\delta_{\mathrm{H}}(400 \mathrm{MHz}, \mathrm{DMSO}):(1 \mathrm{H}, \mathrm{s}) 12.40,(1 \mathrm{H}, \mathrm{t})$ $8.35,(1 \mathrm{H}, \mathrm{t}) 7.97,(1 \mathrm{H}, \mathrm{t}) 7.74,(1 \mathrm{H}, \mathrm{t}) 7.66,(1 \mathrm{H}, \mathrm{m}) 7.54,(1 \mathrm{H}, \mathrm{m}) 7.47(1 \mathrm{H}, \mathrm{m}) 7.36,(1 \mathrm{H}, \mathrm{m})$ 7.24, $(1 \mathrm{H}, \mathrm{s}) 5.18,(1 \mathrm{H}, \mathrm{s}) 4.12,(1 \mathrm{H}, \mathrm{s}) 3.85,(1 \mathrm{H}, \mathrm{s}) 3.70$; Elemental Analysis: Cal. C, 56.3; H, 3.42; N, $10.96 \%$. Found. C, 56.31; H, 3.40; N, $10.89 \%$.

\section{3-(4-chlorophenyl)-2-(2,4-dihydroxyquinolin-3-yl)-1,3-thiazolidin-4-one $\left(\mathrm{SH}_{\mathrm{e}}\right)$}

Off-white colored solid compound, M.P. $>300{ }^{\circ} \mathrm{C}$, Yield 755; Spectral data: IR (KBr) $1160.00 \mathrm{~cm}^{-1}, 1618.79 \mathrm{~cm}^{-1}, 1669.03 \mathrm{~cm}^{-1}, 3081.00 \mathrm{~cm}^{-1}, 870.18 \mathrm{~cm}^{-1}$; Mass: $372.03(\mathrm{M})^{+} ;{ }^{1} \mathrm{H}$ NMR: $\delta_{\mathrm{H}}(400 \mathrm{MHz}, \mathrm{DMSO}):(1 \mathrm{H}, \mathrm{s}) 12.43,(1 \mathrm{H}, \mathrm{t}) 8.18,(1 \mathrm{H}, \mathrm{t}) 7.88,(1 \mathrm{H}, \mathrm{t}) 7.79,(1 \mathrm{H}, \mathrm{t})$ 7.67, $(1 \mathrm{H}, \mathrm{m}) 7.60,(1 \mathrm{H}, \mathrm{m}) 7.45(1 \mathrm{H}, \mathrm{m}) 7.33,(1 \mathrm{H}, \mathrm{m}) 7.20,(1 \mathrm{H}, \mathrm{s}) 5.13,(1 \mathrm{H}, \mathrm{s}) 3.89,(1 \mathrm{H}$, s) $3.79,(1 \mathrm{H}, \mathrm{s}) 3.70$; Elemental Analysis: Cal. C, 57.99; H, 3.51; N, 7.51\%. Found. C, 57.94; $\mathrm{H}, 3.53 ; \mathrm{N}, 7.58 \%$.

\section{BIOLOGICAL EVALUATION}

All newly synthesized quinoline analog azetidin and thiazolidin derivatives $\left(\mathrm{SH}_{1-5}\right.$ and $\mathrm{SH}_{\mathrm{a}-\mathrm{e}}$ ) were examined for antibacterial activity against two gram positive bacterial strains (Staphylococcus aureus MTCC 96, Bacillus cereus MTCC 430), two gram negative bacterial strains (Pseudomonas aeruginosa MTCC 741, Klebsiella pneumoniae MTCC 109) and two fungal strains (Aspergillus clavatus MTCC 1323, Candida albicans MTCC 227 and Aspergillus niger MTCC 282) using the agar dilution method [21].

Here, Gentamycin, Ampicillin and Chloramphenicol were used as a standard control drug for antibacterial activity, whereas Nystatin and Greseofulvin were used as a standard control drug for antifungal activity. To determine the minimum inhibitory concentration, a stock solution of the final synthesized compounds $(2000 \mu \mathrm{g} / \mathrm{mL})$ was prepared in dimethyl sulfoxide and then test compounds were incorporated in a specified quantity of molten sterile agar, i.e., nutrient agar and dextrose agar for antibacterial and for antifungal screening respectively.

\section{1. In vitro antibacterial activity}

\section{1. 1. Determination of minimal inhibitory concentration (MIC)}

The sterile test tubes containing $1 \mathrm{ml}$ of sterile media were added to $1 \mathrm{ml}$ of different serially diluted test samples. To these tubes, $0.1 \mathrm{ml}$ of suspension of respective microorganism was added in normal saline and incubated at $37 \pm 2{ }^{\circ} \mathrm{C}$ for $24 \mathrm{hr}$. After $24 \mathrm{hr}$ a loop full of samples was streaked in zigzag fashion over the agar medium in a petri plate from the culture and this was incubated at $37 \pm 2{ }^{\circ} \mathrm{C}$ for 24 hours. Then the lowest concentration of the sample that inhibited the microbial growth in the petri dish was determined and this is considered as MIC. This procedure was performed to confirm the MIC. The results are summarized in Table 1. 
Table 1. Result of antibacterial by MIC method of the synthesized compounds.

\begin{tabular}{|c|c|c|c|c|c|}
\hline \multicolumn{6}{|c|}{ Minimal inhibition concentration $[\boldsymbol{\mu g} / \mathbf{m L}]$} \\
\hline $\begin{array}{c}\text { Sr. } \\
\text { no. }\end{array}$ & Code no. & $\begin{array}{c}\text { E.Coli } \\
\text { MTCC 443 }\end{array}$ & $\begin{array}{c}\text { P. Aeruginosa } \\
\text { MTCC } 1688\end{array}$ & $\begin{array}{c}\text { S. Aureus } \\
\text { MTCC 96 }\end{array}$ & $\begin{array}{c}\text { S. Pyogenus } \\
\text { MTCC 442 }\end{array}$ \\
\hline Std. & Ampicillin & 100 & 100 & 250 & 100 \\
\hline Std. & Chloramphenicol & 50 & 50 & 50 & 50 \\
\hline 1 & SH 1 & 250 & 100 & 250 & 100 \\
\hline 2 & SH 2 & 125 & 62.5 & 125 & 50 \\
\hline 3 & SH 3 & 50 & 125 & 250 & 100 \\
\hline 4 & SH 4 & 62.5 & 200 & 62.5 & 125 \\
\hline 5 & SH 5 & 125 & 100 & 100 & 250 \\
\hline 6 & SH a & 200 & 125 & 125 & 100 \\
\hline 7 & SH b & 125 & 100 & 200 & 125 \\
\hline 8 & SH c & 62.5 & 200 & 125 & 200 \\
\hline 9 & SH d & 100 & 200 & 62.5 & 250 \\
\hline 10 & SH e & 125 & 100 & 100 & 250 \\
\hline
\end{tabular}

\section{2. In vitro antifungal activity}

\section{2. 1. Determination of minimal inhibitory concentration (MIC):}

Table 2. Result of antifungal activity for the synthesized compounds.

\begin{tabular}{|c|c|c|c|c|}
\hline \multicolumn{5}{|c|}{ Minimal concentration inhibition $[\mu \mathrm{g} / \mathrm{mL}]$} \\
\hline Sr. no. & Code no. & $\begin{array}{l}\text { C. Albicans } \\
\text { MTCC } 227\end{array}$ & $\begin{array}{c}\text { A. Niger } \\
\text { MTCC } 282\end{array}$ & $\begin{array}{l}\text { A. Clavatus } \\
\text { MTCC } 1323\end{array}$ \\
\hline 1 & Nystatin & 100 & 100 & 100 \\
\hline 2 & Greseofulvin & 500 & 100 & 100 \\
\hline 3 & $\mathrm{SH}_{1}$ & 500 & 1000 & 1000 \\
\hline 4 & $\mathrm{SH}_{2}$ & 1000 & 500 & 500 \\
\hline 5 & $\mathrm{SH}_{3}$ & 1000 & 500 & 500 \\
\hline 6 & $\mathrm{SH}_{4}$ & 250 & $>1000$ & $>1000$ \\
\hline 7 & $\mathrm{SH}_{5}$ & 500 & $>1000$ & $>1000$ \\
\hline 8 & $\mathrm{SH}_{\mathrm{a}}$ & 100 & $>1000$ & $>1000$ \\
\hline 9 & $\mathrm{SH}_{\mathrm{b}}$ & 1000 & 1000 & 1000 \\
\hline 10 & $\mathrm{SH}_{\mathrm{c}}$ & $>1000$ & 500 & 500 \\
\hline 11 & $\mathrm{SH}_{\mathrm{d}}$ & 500 & 1000 & $>1000$ \\
\hline 12 & $\mathrm{SH}_{\mathrm{e}}$ & 500 & $>1000$ & $>1000$ \\
\hline
\end{tabular}


The inoculums were prepared by taking a loopful of stock culture to about $100 \mathrm{~mL}$ of nutrient broth, in $250 \mathrm{~mL}$ clean and sterilized conical flasks. The flasks were incubated at 27 ${ }^{\circ} \mathrm{C}$ for $24 \mathrm{hr}$ before use. The plates were kept undisturbed for at least two hours at room temperature toallow diffusion of the solution properly, into potato-dextrose-agar medium. Then theplates were incubated at $25{ }^{\circ} \mathrm{C}$ for $48 \mathrm{hr}$. The highest dilution showing at least $99 \%$ inhibition zone is taken as MIC. The result of this is much affected by the size of the Inoculum. The experiments were performed in triplicate in order to minimize the errors. The results of antifungal activity are given in the Table 2 .

\section{RESULTS AND DISCUSSION}

\section{1. Chemistry}

The title compounds, quinoline analoge azetidin and thiazolidin derivatives (Table 1) were synthesized via four step reactions starting from the 4-Hydroxyquinolin-2(1H)-one $\left(\mathrm{S}_{\mathrm{A}}\right)$. Here, 4-Hydroxyquinolin-2(1H)-one firstly undergoes Reimer-Tiemann formaylation reaction in the presence of sodium hydroxide and chloroform to afforded 4-Hydroxy-3 formylquinolin-2(1H)-one $\left(\mathrm{S}_{\mathrm{B}}\right)$ in good yield. This formaylated compound condensed with various aromatic amines in the presence of acetic acid to afforded various schiff-base in excellent yields $\left(\mathrm{S}_{1-5}\right)$. (Scheme1). Finally these Schiff-base were incorporated in the synthesis of azetidin $\left(\mathrm{SH}_{1-5}\right)$ and thiazolidin $\left(\mathrm{SH}_{\mathrm{a}-\mathrm{e}}\right)$ derivatives by using chloroacetyl chloride and thioglycolic acid respectively. The structures of products $\left(\mathrm{SH}_{1-5}\right)$ and $\left(\mathrm{SH}_{\mathrm{a}-\mathrm{e}}\right)$ were confirmed by different spectroscopic techniques like IR, ${ }^{1} \mathrm{H}$ NMR, Mass and elemental analysis. Both an electron withdrawing and electron donating substituents on the aromatic ring afforded excellent yield of the products. Both the azetidin $\left(\mathrm{SH}_{1-5}\right)$ and thiazolidin $\left(\mathrm{SH}_{\mathrm{a}-\mathrm{e}}\right)$ derivatives were prepared under microwave irradiation, which facile the conversion of the reaction within short period of time compared to conventional procedure.

\section{2. Antibacterial activity}

The antibacterial activity of the synthesized compounds $\mathrm{SH}_{1-5}$ and $\mathrm{SH}_{\mathrm{a}-\mathrm{e}}$ was determined in-vitro using MIC (Broth Dilution Method) against four pathogenic micro-organisms viz. $E$. coli, $P$. aeruginosa $(\mathrm{Gm}-\mathrm{ve})$ and $S$. aureus, S. pyogenus $(\mathrm{Gm}+\mathrm{ve})$ at various concentrations. Table 2 shows that all the newly synthesized quinoline scaffolds were found to exhibit good to moderate activity against the specific microbial strain, among which, $\mathrm{SH}_{4}$ and $\mathrm{SH}_{\mathrm{c}}$ exhibited high potent activity against $E$. coli at $\mathrm{MIC}$ of $62.5 \mu \mathrm{g} / \mathrm{ml} . \mathrm{SH}_{\mathrm{d}}$ and $\mathrm{SH}_{3}$ shows activity against $E$. Coli equivalent to Ampicillin and Chloramphenicol respectively. $\mathrm{SH}_{2}$, shows equipotent activity against $P$. aeruginosa while $\mathrm{SH}_{1}, \mathrm{SH}_{5}, \mathrm{SH}_{\mathrm{b}}$ and $\mathrm{SH}_{\mathrm{e}}$ showed activity against $P$. Aeruginosa at MIC of $100 \mu \mathrm{g} / \mathrm{mL}$, which is equivalent to standard drug ampicillin.

All synthesized compounds showed good activity against $S$. aureus, however $\mathrm{SH}_{4}$ and $\mathrm{SH}_{\mathrm{d}}$ exhibited best potent activity against $S$. aureus. $\mathrm{SH}_{2}$ shows activity against $S$. pyogenus at MIC of $50 \mu \mathrm{g} / \mathrm{mL}$ which is equivalent to standard drug Chloramphenicol. While $\mathrm{SH}_{1}, \mathrm{SH}_{3}$ and $\mathrm{SH}_{\mathrm{a}}$ shows potent activity against $S$. Pyogenus at MIC of $100 \mu \mathrm{g} / \mathrm{mL}$, which is equivalent to standard drug ampicillin. All the remaining final quninoline derivatives were found to demonstrate good-to-poor activity profiles at minimum inhibitory concentration levels. 


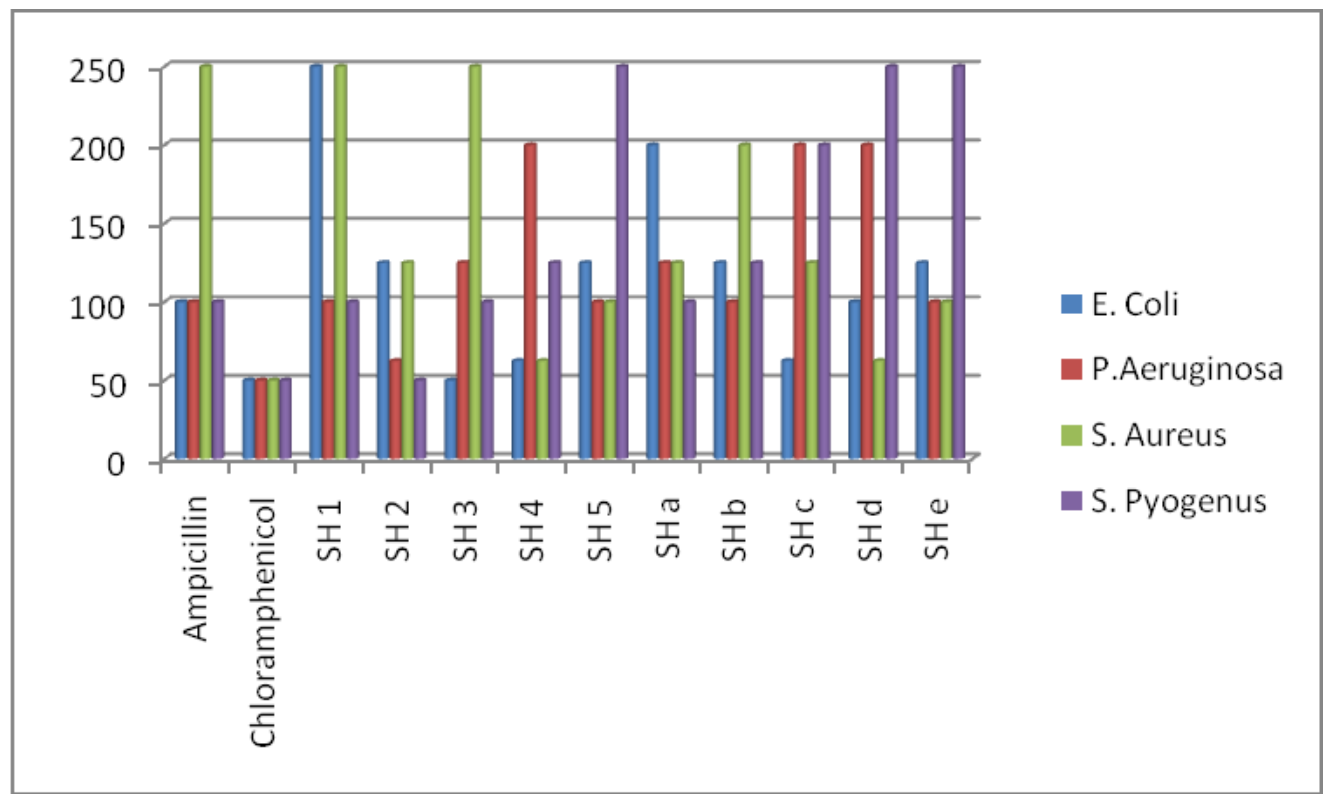

\section{3. Antifungal activity}

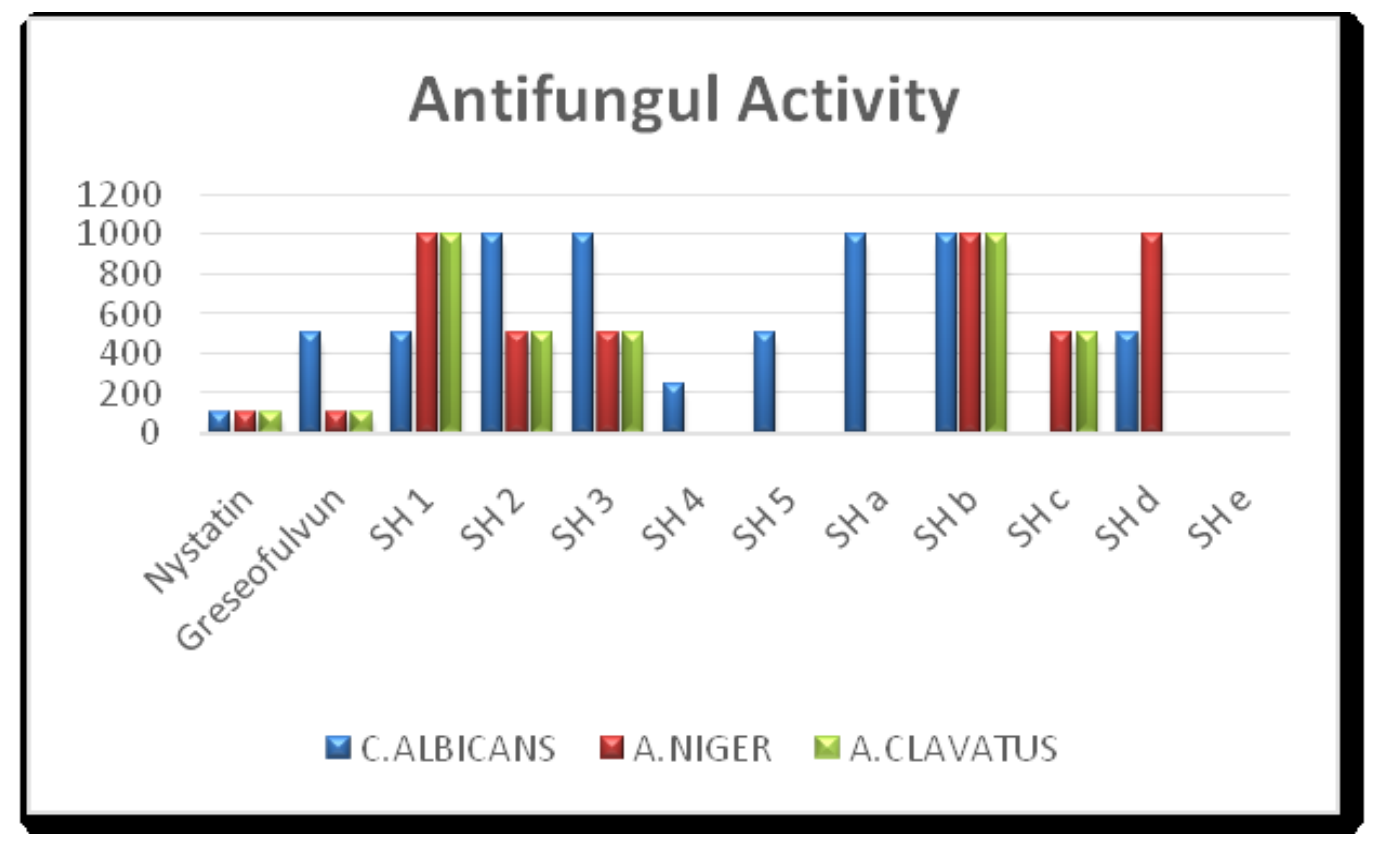

The antifungal activity of the synthesized compounds $\mathrm{SH}_{1-5}$ and $\mathrm{SH}_{\mathrm{a}-\mathrm{e}}$ was determined in-vitro using an Agar plate method against three strains viz. C. albicans, A.niger and A.clavatus at different concentration ranging between $100 \mu \mathrm{g} / \mathrm{ml}$ to $1250 \mu \mathrm{g} / \mathrm{ml}$. Out of the tested compounds $\mathrm{SH}_{4}$ exhibited a significant activity against C.albicans at concentration of $250 \mu \mathrm{g} / \mathrm{ml}$ reference standard Griseofulvin at concentration $500 \mu \mathrm{g} / \mathrm{mL}$. SH $\mathrm{SH}_{1}, \mathrm{SH}_{5}, \mathrm{SH}_{\mathrm{d}}$ and $\mathrm{SH}_{\mathrm{e}}$ shows equipotent antifungal activity at MIC $500 \mu \mathrm{g} / \mathrm{ml}$. 


\section{CONCLUSION}

In this article, we have elaborated the initial efforts made toward the sighting of novel, potentially active quinoline analog two novel series of azetidin $\left(\mathrm{SH}_{1-5}\right)$ and thiazolidin $\left(\mathrm{SH}_{\mathrm{a}-\mathrm{e}}\right)$ derivatives which were synthesized by simple and efficient method. From the bioassay it is clear that the introduction of appropriate halo, nitro and the methoxy substituent on the phenyl ring would lead to the more active antimicrobial derivatives. Compounds were screened against wide range of pathogenic bacteria and fungal strains shows better activity. Overall, from the bioassay results, we just conclude that findings of the present study will have a good impact on medicinal chemists to synthesize similar analogous which will show enhanced bioactivity.

\section{Acknowledgements}

The authors are thankful to the Department of Chemistry, Gujarat University Ahmedabad, for providing the necessary facilities. UGC-Info net \& INFLIBNET Gujarat University are acknowledged for providing the esource facilities. One of us R.H.V. is thankful to UGC-BSR (F.7-74/2007 (BSR)) for financial assistance.

\section{References}

[1] U. Desai, S. Mitragotri, T. Thopate, D. Pore, P. Wadgaonkarb, Arkivoc 15 (2006) 198204.

[2] E.E. Ebenso, M.M. Kabanda, T. Arslan, M. Saracoglu, F. Kandemirli, L. C. Murulana, A. K. Singh, S. K. Shukla, B. Hammouti, K. Khaled, Int. J. Electrochem. Sci. 7 (2012)

[3] S. Bawa, S. Kumar, S. Drabu, R. Kumar, J. Pharm. Bioallied. Sci. 2 (2010) 64-71.

[4] M. Ozyanik, S. Demirci, H. Bektas, N. Demirbas, A. Demirbas, S.A. Karaoglu, Turk. J. Chem. 36 (2012) 233-246.

[5] P.R. Graves, J.J. Kwiek, P. Fadden, R. Ray, K. Hardeman, A.M. Coley, M. Foley, T.A. Haystead, Mol. Pharmacol. 62 (2002) 1364-1372.

[6] N. Ahmed, K.G. Brahmbhatt, S. Sabde, D. Mitra, I. Singh, K. K. Bhutani, Bioorg. Med. Chem. 18 (2010) 2872-2879.

[7] D.K. Shukla, S.D. Srivastava, Indian J. Chem. 47B (2008) 463.

[8] T.R. Rawat, S.D. Srivastava, Indian J. Chem. 37B (1998) 91.

[9] Nema, S.K. Srivastava, J. Indian Chem. Soc. 84 (2007) 1037.

[10] V.V. Mulwad, A.A. Mir, J. Korean Chem. Soc. 52 (2008) 649.

[11] A.K. Parikh, P.S. Oza, S.B. Bhatt, Indian J. Chem. 44B (2005) 585.

[12] R.B. Patel, P.S. Desai, K.H.Chikhalia, Indian J. Chem. 45B (2006) 773.

[13] S.K. Srivastava, S. Srivastava, S.D. Srivastava, Indian J. Chem. 38B (2000) 464.

[14] S.K. Srivastava, S.L. Srivastava, S.D. Srivastava, Indian J. Chem. 39B (1999) 183.

[15] E. Alonsodel, C. Pozo, J. Gonzalez, Synlett. 1 (2002) 69.

[16] J.W. Skiles, D. McNeil, Tetrahedron Lett. 31 (1990) 7277. 
[17] Nefzi, J.M. Ostresh, R.A. Houghten, Chem. Rev. 97 (1997) 449.

[18] R.G. Frazen, J. Comb. Chem. 2 (2000) 195.

[19] M.L. Barreca, E.D. Clercq, J. Med. Chem. 45 (2002) 5410.

[20] T. Takematsu, K. Yokoyama, K. Ikeda, Y. Haashi, E. Taniyama, Japanese Patent 75 (1975) 121.

[21] T. Zsolnai, Acta Phytopathol. Acad. Sci. Hung. 9 (1974) 125.

[22] C.R. Harris, S.A.Turnbull, Can. Entomol. 109 (1977) 1109.

[23] J. Mohan, V.K. Chadha, H.S. Chaudhary, B.D. Sharma, H.K. Pujari, L.N. Mohapatra, Ind. J. Exp. Biol. 10 (1972) 37.

[24] E.B. Akerblom, J. Med. Chem. 17 (1974) 609.

[25] Dandia, R. Singh, S. Khaturia, C. Merienne, G. Morgantc, A. Loupyd, Bioorg. Med. Chem. 14 (2006) 2409.

[26] R. Ottana, E. Mazzon, L. Dugo, F. Monforte, R. Maccari, L. Sautebin, G. Deluca, M.G. Vigorita, S. Alcaro, F. Ortuso, A.P. Caputi, S. Cuzzocrea, Eur. J. Pharmacol. 448 (2002) 71.

[27] Goel, A. Kumar,Eur. J. Med. Chem. 34 (1999) 265.

[28] R. Ottana, R. Maccari, M.G. Bruno. A. Rotondo, A. Rossi, G. Chiricosta, R.D. Paola, L. Sautebin, S. Cuzzocread, M.G. Vigorita, Bioorg.Med. Chem. 13 (2005) 4243.

[29] N. Karali, A. Kocabalkanli, A. Gursoy, O. Ates, II Farmaco 57 (2002) 589.

[30] T. Srivastava, A.K. Gaikwad, W. Haq, S. Sinha, S.B. Katti, Arkivoc. 2 (2005) 120.

[31] M.L. Barreca, Bioorg. Med. Chem. Lett. 11 (2001) 1793.

[32] V. Gududuru, Bioorg. Med. Chem. Lett. 14 (2004) 5289.

[33] S.M. Prajapati, K.D. Patel, R.H. Vekariya, S.N. Panchal, H. D. Patel, RSC Adv. 4 (2014) 24463-24476.

[34] S.J. Wadher, M.P. Puranik, N.A. Karande, P.G. Yeole, Int. J. Pharm. Tech. Res. 1 (2009) 22-33.

[35] S.B. Desai, P.B. Desai, K.R. Desai, Heterocycl. Commun. 7 (2001) 83-90. 